\title{
Hôtels et dépendances
}

Les femmes de chambre en Europe

Housekeepers in various European countries

\section{Christine Guégnard et Sylvie-Anne Mériot}

\section{CpenEdition}

Journals

Édition électronique

URL : http://journals.openedition.org/travailemploi/1617

DOI : 10.4000/travailemploi.1617

ISSN : 1775-416X

Éditeur

DARES - Ministère du Travail

Édition imprimée

Date de publication : 15 mars 2010

Pagination : 55-66

ISSN : 0224-4365

Référence électronique

Christine Guégnard et Sylvie-Anne Mériot, «Hôtels et dépendances », Travail et Emploi [En ligne],

121 | janvier-mars 2010, mis en ligne le 15 mars 2011, consulté le 01 mai 2019. URL : http:// journals.openedition.org/travailemploi/1617 ; DOI : 10.4000/travailemploi.1617 


\title{
Hôtels et dépendances: les femmes de chambre en Europe
}

\author{
Christine Guégnard(*), Sylvie-Anne Mériot(**)
}

Cet article présente une analyse de la qualité de l'emploi des femmes de chambre dans divers pays d'Europe sous le double aspect des contraintes patronales de gestion de main-d'œuvre, et de la construction des trajectoires professionnelles des salariées. Vulnérables, peu qualifiées et peu valorisées, souvent d'origine étrangère, la plupart des femmes de chambre sont aisément fidélisées du fait de leur faible employabilité sur le marché du travail. En réalité, elles se trouvent reléguées dans une spirale qui lie flexibilité, rémunération médiocre, précarité plurielle, renforcée par les pratiques de dépendance des hôteliers.

La persistance des emplois non qualifiés dans des pays européens où le niveau d'éducation ne cesse de s'élever interpelle. S'agit-il d'un point de passage plus ou moins inévitable mais transitionnel, ou demeure-t-il un emploi durable sans perspective pour une main-d'œuvre captive, comme le suggère la théorie du marché dual du travail? L'Institut de recherche sur l'éducation, sociologie et économie de l'éducation, et le Centre d'études et de recherches sur les qualifications ont participé à une recherche comparative au niveau européen sur le travail peu qualifié dans l'hôtellerie. Ce projet impulsé par la Russell Sage Foundation faisait suite à une recherche conduite aux États-Unis sur la thématique du Low-Wage Work (cf. encadré 1). Il a été développé parallèlement selon une méthodologie élaborée en commun dans cinq pays - Allemagne, Danemark, France, Pays-Bas, Royaume-Uni - pour cinq emplois cibles dont les femmes de chambre de l'hôtellerie. L'objectif était d'identifier les stratégies de management des entreprises, d'analyser les situations d'emploi et les perspectives de ces salariées situées en bas de la hiérarchie des qualifications et des rémunérations dans divers hôtels d'Europe. Il s'agit de comprendre les contrastes et les similitudes pour un même métier exercé dans un secteur identique, tant en termes de conditions de travail, de contraintes d'activités et d'opportunités, dans des contextes sociaux, institutionnels et économiques distincts: la qualité d'un emploi peu qualifié diffère-t-elle d'un pays à l'autre? Telle est la question à laquelle nous allons tenter de répondre en prenant comme exemple les femmes de chambre.

(*) Institut de recherche sur l'éducation, sociologie et économie de l'éducation - IREDU/CNRS, Céreq, université de Bourgogne; christine.guegnard@u-bourgogne.fr

$(* *)$ Centre d'études et de recherches sur les qualifications; sa.meriot@free.fr
La chambre est au cœur du business de l'hôtellerie et la qualité du nettoyage a une importance stratégique dans le succès des hôtels. Or, l'activité des femmes de chambre repose sur l'invisibilité. Leur emploi est souvent défini par la négative: pas ou peu qualifié, caractérisé par des tâches répétitives et exécutées sous le contrôle direct d'une hiérarchie, pas de référence au diplôme et à l'expérience, peu de prise d'autonomie ou de responsabilité, pas ou peu d'évolution technologique, peu de perspective professionnelle. Fortement dépendantes des entreprises du fait de leur statut et contrat de travail, subordonnées aux stratégies de leur employeur, fragilisées de par leurs caractéristiques personnelles, les femmes de chambre cumulent précarité, intensification du travail, disponibilité temporelle, faiblesse des rémunérations.

Cet article analyse la qualité de l'emploi dans cinq pays d'Europe sous le double aspect des contraintes patronales de gestion de main-d'œuvre, et de la construction des trajectoires professionnelles de ces salariées. Les conditions d'emploi au regard des stratégies des employeurs constituent le cœur de la première partie. Sont ainsi identifiés les traits principaux et les tendances communes de l'hôtellerie qui se distingue des autres activités économiques à plus d'un titre. Au fil des ans, si les chaînes supplantent les hôtels indépendants, l'hôtellerie relève en majorité d'une gestion de petites unités largement indépendantes dans lesquelles les organisations représentatives du personnel sont peu implantées. Les rémunérations proposées figurent parmi les plus basses dans chaque contrée, les femmes de chambre se situant en bas de l'échelle, souvent payées à temps partiel ou sur des contrats précaires et courts. Cette section souligne avant tout le rôle des institutions et des entreprises pour maintenir ces emplois dépendants du segment secondaire (Doeringer, Piore, 1971) en référence à la théorie du marché dual; le 


\section{Encadré 1}

\section{Une recherche sur les emplois à bas salaires}

Cet article s'inscrit dans le prolongement d'une recherche portant sur la gestion des emplois à bas salaire, impulsée par la Russell Sage Foundation. Depuis 2004, l'Institut de recherche sur l'éducation, sociologie et économie de l'éducation (IREDU-CNRS) et le Centre d'études et de recherches sur les qualifications ont participé à cette recherche comparative au niveau européen sur l'avenir du travail peu qualifié dans l'hôtellerie. Le projet, coordonné en France par le Centre d'études prospectives d'économie mathématique appliquée à la planification, faisait suite à une recherche conduite aux États-Unis sur la thématique du Low-Wage Work (APPelBAUm et al.). II a été développé selon la même méthodologie élaborée en commun dans quatre autres pays d'Europe: Allemagne, Danemark, Pays-Bas, Royaume-Uni. Ces pays ont été choisis par la Russell Sage Foundation en fonction de leur niveau de développement économique similaire, leur histoire politique et institutionnelle, leurs caractéristiques géographiques.

Cinq emplois ont été ciblés dans cinq secteurs d'activité précis: les femmes de chambre de l'hôtellerie, les aides-soignants et les agents de service dans les hôpitaux, les opérateurs des industries agro-alimentaires, les personnels de caisse et les vendeurs dans la grande distribution, les téléopérateurs des centres d'appel. Tous ces métiers sont situés au bas de la hiérarchie des qualifications et des rémunérations aux États-Unis, et l'objectif était de comparer entre pays les stratégies de management des employeurs, le travail, les conditions d'emploi et les perspectives de ces salariés peu qualifiés. Les premiers résultats ont été publiés par la Russell Sage Foundation en 2008 sous la forme d'un ouvrage par pays (pour la France cf. CAROLI, GAUTIÉ, 2008; 2009).

Pour réaliser cette recherche menée entre 2004 et 2008 en France, nous avons combiné des approches quantitatives et qualitatives:

- une étude de documents et de statistiques issus des enquêtes de l'Insee (emploi, déclarations annuelles de données sociales, enquête annuelle d'entreprise, conditions de travail) et de la Direction du tourisme, des statistiques des Assedic, Unedic, d'Eurostat, de l'Union des métiers et des industries de l'hôtellerie, du groupe Accor, des bases de données du Céreq;

- une analyse d'enquêtes de terrain avec des interviews de salariés (une soixantaine dont des femmes de chambre, des gouvernantes, des responsables d'hôtels), et des entretiens avec des cadres de sièges sociaux et représentants de syndicats ou d'organisations patronales. Huit hôtels ont été sélectionnés sur des critères fixés en commun avec les autres équipes: la localisation (4 dans la capitale, 4 en province), la catégorie (4 hôtels économiques $2^{*}, 4$ hôtels $3-4^{*}$ et plus), le statut (4 indépendants, 4 appartenant à des chaînes dont Accor) et la taille des établissements (incluant des hôtels de moins de vingt salariés).

Les analyses présentées dans cet article proviennent à la fois des travaux et des entretiens menés en France par GuéGnard et al. (2005); GuÉGnard, MÉriot (2007, 2008); Eriksonn, Li (2008); Hermanusssen (2008); Vanselow (2008) et des travaux produits par les équipes des quatre pays européens et rédigés par DutTon et al., ERIKSSON et LI, Hermanussen, Vanselow (2008).

La qualité de l'emploi est un concept multidimensionnel qui pose de nombreuses questions théoriques et empiriques présentées par DavoINE et ERHEL (2007). La qualité de l'emploi des femmes de chambre est ici appréhendée par diverses composantes dont le salaire, la stabilité du contrat, la durée et le temps de travail, la formation, la carrière, la conciliation des vies professionnelle et familiale.

marché primaire se caractérise par de bonnes conditions de travail, une stabilité, des chances de promotion, de hauts salaires, contrairement aux emplois du marché secondaire. La deuxième partie met davantage l'accent sur les conditions de travail et dévoile les caractéristiques d'une population singulière par rapport aux autres salariés de l'hôtellerie, particulièrement exposée aux risques de précarité. Une vision comparative, de la qualité de l'emploi des femmes de chambre et de leurs modes de vie au quotidien, apporte un éclairage spécifique sur la segmentation du marché du travail (PIORE, 1978). Isolées, vulnérables, souvent d'origine étrangère, la plupart des salariées sont aisément fidélisées du fait de leur faible employabilité sur le marché du travail. Il leur est difficile de sortir de la spirale qui lie flexibilité, précarité plurielle, rémunération modique. En réalité, un dualisme multiforme apparaît dans l'hôtellerie renforçant ainsi la dépendance du personnel, surtout en France.

\section{Des spécificités analogues entre pays}

S'appuyer sur des comparaisons européennes pour analyser les spécificités des travailleurs à bas salaire ne peut se faire sans dessiner un portrait de l'hôtellerie qui présente des traits comparables dans les cinq pays. Ce secteur dynamique, mais peu rémunérateur, se distingue des autres activités par une prédominance des indépendants ou des nonsalariés, une importance des postes d'exécution, la jeunesse et la féminisation de la main-d'œuvre, la faiblesse du dialogue social, et une pratique syndicale rare qui contraste avec d'influentes unions patronales.

\section{Un secteur typique}

L'activité hôtelière est fortement soumise aux variations saisonnières, corrélée aux évolutions 
Tableau 1 : Le monde de l'hôtellerie dans cinq pays d'Europe

\begin{tabular}{|l|c|c|c|c|c|}
\hline & Allemagne & Danemark & France & Pays-Bas & Royaume-Uni \\
\hline Nombre de touristes & 57100000 & 2840000 & 31500000 & 9100000 & 30200000 \\
\hline Nombre d'hôtels & 36200 & 470 & 18140 & 3100 & 39330 \\
\hline Nombre de lits & 1632100 & 69000 & 1254000 & 192000 & 1223000 \\
\hline $\begin{array}{l}\text { Personnes occupées en héberge- } \\
\text { ment touristique }\end{array}$ & 375000 & 22000 & 256000 & 72000 & 309000 \\
\hline Part des femmes & $69 \%$ & $63 \%$ & $55 \%$ & $61 \%$ & $56 \%$ \\
\hline Part des temps partiels & $32 \%$ & $44 \%$ & $22 \%$ & $60 \%$ & $40 \%$ \\
\hline $\begin{array}{l}\text { Part des hôtels de moins de dix } \\
\text { salariés }\end{array}$ & $80 \%$ & $70 \%$ & $80 \%$ & $65 \%$ & $64 \%$ \\
\hline $\begin{array}{l}\text { Taux de syndicalisation } \\
\text { - au sein de l'hôtellerie }\end{array}$ & $23 \%$ & $80 \%$ & $8 \%$ & $26 \%$ & $29 \%$ \\
\end{tabular}

Sources : Eurostat (2006-2007), rapports européens Low-Wage Work, Russell Sage Foundation (2005-2006).

conjoncturelles de chaque pays, les événements politiques ou monétaires internationaux provoquant aussi des fluctuations d'activité, particulièrement marquantes ces dernières années. Malgré ces aléas, l'hébergement touristique demeure dynamique dans les pays étudiés. En termes d'emploi, il occupe plus de deux millions de personnes en Europe, les effectifs les plus importants se situent au Royaume-Uni et en Allemagne, suivis par la France ( $c f$. tableau 1). La France possède la quatrième capacité hôtelière européenne après l'Italie, l'Allemagne et l'Espagne et devant le Royaume-Uni. Depuis une quinzaine d'années, le développement de l'hôtellerie de chaîne a modifié le paysage dans les cinq territoires enquêtés. Malgré cette progression continue, les indépendants demeurent majoritaires en nombre d'établissements et en capacité d'accueil. Près de $80 \%$ des hôtels sont indépendants, et $64 \%$ à $80 \%$ des établissements comptent moins de dix salariés selon le pays étudié. $\mathrm{Ce}$ sont des petites entreprises de gestion indépendante, souvent familiales comme les bed and breakfast fortement développés au Royaume-Uni.

Plusieurs points sont communs aux cinq secteurs d'activité. Ils offrent une porte d'entrée sur le marché du travail pour de nombreux jeunes (et des étudiants), des immigrés ou des étrangers, notamment sur des postes nécessitant de faibles qualifications. De plus, les femmes y jouent traditionnellement un rôle, représentant plus de la moitié des effectifs : de $55 \%$ en France à $69 \%$ en Allemagne, la moyenne européenne étant de $60 \%$. Et quel que soit le territoire, les réglementations ne sont pas toujours respectées dans la myriade des petits hôtels, les employeurs étant, selon leurs propres termes, doués pour ignorer la loi ou trouver les moyens de la contourner. Le travail au noir, difficilement mesurable, est également un fait plus ou moins évoqué dans les cinq contrées, qui peut correspondre à du travail non déclaré ou partiellement déclaré, des heures supplémentaires non payées, une dissimulation des horaires effectués...

\section{Le salaire de la peine}

Autre similitude, l'hôtellerie est dans les cinq pays le secteur emblématique des travailleurs à bas salaires. Les travailleurs à bas salaire sont définis comme les personnes qui perçoivent moins des deux tiers du salaire médian de leur pays, selon la définition de l'Ocde. La proportion des actifs embauchés à bas salaire varie de $9 \%$ au Danemark à $22 \%$ en Allemagne ou au Royaume-Uni (CAROLI, GAUTIÉ, 2008). Or, ces chiffres sont nettement plus élevés pour l'hôtellerie: le Danemark, la France et les Pays-Bas comptent plus du quart de personnes à bas salaire, le Royaume-Uni plus de la moitié, l'Allemagne plus des deux tiers (1) ( $c f$. tableau 2).

Alors que la chambre est au cœur du service, la faiblesse des rétributions reflète la place subalterne attribuée aux femmes de chambre, en bas de l'échelle. Les rémunérations sont ajustées sur le salaire national minimum légal en France(2), aux Pays-Bas et Royaume-Uni, le minimum tarif au Danemark, et le salaire de base négocié aux plans national et régional en Allemagne. Le salaire net déclaré par une femme de chambre varie de 7 à 9 euros selon les pays, excepté au Danemark, où il oscille entre 13 et 15 euros, bien en dessous du salaire horaire danois (de 29,70 euros tous métiers confondus). Dans quelques hôtels de luxe français ou britanniques, la pratique d'autocontrôle par le personnel

(1) Les chiffres calculés sur une base horaire intègrent les apprentis pour les Pays-Bas et Royaume-Uni, ce qui tend à surestimer légèrement ce taux. De plus, aux Pays-Bas et en Allemagne l'importance du temps partiel avec une rémunération horaire plus faible influe sur le taux élevé des travailleurs à bas salaires (CAROLI, GaUtié, 2009).

(2) Ainsi en France, le seuil de bas salaire en 2005 est de 5,92 euros (net par heure), le taux horaire du SMIC est de 6,15 euros net, et le salaire net déclaré par les femmes de chambre varie de 6,37 à 8,71 euros ( $c f$. tableau 2). Rappelons qu'en France, les employeurs bénéficient d'allégements de charges sociales sur les bas salaires, ce qui peut inciter les employeurs à maintenir leur personnel à de bas niveaux de rémunération. 
Tableau 2 : Les travailleurs à bas salaire dans l'hôtellerie

\begin{tabular}{|c|c|c|c|c|c|}
\hline & Allemagne & Danemark & France & Pays-Bas & Royaume-Uni \\
\hline $\begin{array}{l}\text { Femmes de chambre } \\
\text { (effectif ou part dans l'hôtellerie) }\end{array}$ & 28000 & $20 \%$ & $20 \%$ & $25-30 \%$ & 25000 \\
\hline $\begin{array}{l}\text { Part des personnes d'origine étrangère } \\
\text { dans les hôtels }\end{array}$ & $20 \%$ & $60 \%$ & $48 \%$ & $17 \%$ & $60 \%$ \\
\hline Part des non-diplômés & $57 \%$ & $45 \%$ & $64 \%$ & $59 \%$ & $50-60 \%$ \\
\hline $\begin{array}{l}\text { Part des bas salaires: } \\
\text { - dans l'hôtellerie } \\
\text { - dans l'ensemble des salariés }\end{array}$ & $\begin{array}{l}70 \% \\
22 \%\end{array}$ & $\begin{array}{c}25 \% \\
9 \%\end{array}$ & $\begin{array}{l}27 \% \\
10 \%\end{array}$ & $\begin{array}{l}26 \% \\
18 \%\end{array}$ & $\begin{array}{l}59 \% \\
22 \%\end{array}$ \\
\hline $\begin{array}{l}\text { Salaire net horaire déclaré } \\
\text { par les femmes de chambre }\end{array}$ & $\begin{array}{c}7,33 \\
\text { à } 10,36 \text { euros }\end{array}$ & $\begin{array}{c}13 \\
\text { à } 14,70 \text { euros }\end{array}$ & $\begin{array}{c}6,37 \text { à } \\
8,71 \text { euros }\end{array}$ & $\begin{array}{c}7,73 \\
\text { à } 8,72 \text { euros }\end{array}$ & $\begin{array}{c}7,37 \\
\text { à } 8,94 \text { euros }\end{array}$ \\
\hline $\begin{array}{l}\text { Nombre de chambres } \\
\text { à nettoyer par jour }\end{array}$ & $\begin{array}{c}7 \text { à } 18 \\
\text { chambres }\end{array}$ & $\begin{array}{l}14 \text { à } 20 \\
\text { chambres }\end{array}$ & $\begin{array}{l}13 \text { à } 18 \\
\text { chambres }\end{array}$ & $\begin{array}{c}15 \text { à } 21 \\
\text { chambres }\end{array}$ & $\begin{array}{c}9 \text { à } 18 \\
\text { chambres }\end{array}$ \\
\hline Temps par chambre & 15 à 23 minutes & $\begin{array}{l}15 \text { à } 25 \\
\text { minutes }\end{array}$ & 15 à 45 minutes & $\begin{array}{l}15 \text { à } 30 \\
\text { minutes }\end{array}$ & $\begin{array}{l}15 \text { à } 45 \\
\text { minutes }\end{array}$ \\
\hline
\end{tabular}

Sources : rapports européens Low-Wage Work, Russell Sage Foundation (2005-2006). Les travailleurs à bas salaire sont définis comme les personnes qui perçoivent moins des deux tiers du salaire horaire médian de leur pays (apprentis inclus pour les Pays-Bas et Royaume-Uni).

leur permet de gagner davantage: l'autocontrôle est proposé aux femmes de chambre expérimentées, afin qu'elles exécutent elles-mêmes les vérifications à l'aide d'une liste de tâches ou d'objectifs à remplir. Cette méthode s'accompagne d'une prime sur la base de simples vérifications aléatoires et ultérieures de la gouvernante ou du responsable d'hôtel (chaque tâche correctement effectuée est créditée d'un ou deux points qui sont convertis en euros ou en livres en fin de mois). Mais au final, ces rémunérations sont moins élevées que dans la plupart des autres secteurs, pour une part en raison de la faible qualification des emplois; et elles sont proches de celles du commerce de détail et des services personnels (BEAUvors, 2003; Caroli, Gautié, 2009). De plus, dans l'hôtellerie économique, où les rythmes de travail sont les plus intenses, le personnel en France perçoit en moyenne entre 1,5 et 2 euros nets par chambre entretenue, ce qui correspond au prix d'un café!

La majorité des employeurs interviewés retiennent comme base salariale un forfait calculé en fonction du nombre de chambres entretenues, indépendamment des temps effectués et des réglementations en vigueur. Cette pratique prend une acuité particulière dans le cas de la sous-traitance, où l'employeur, est plus distant encore du lieu de travail et de ses salariés. Dans ce contexte, le poids de la masse salariale est perçu comme le seul levier de diminution des coûts, au risque de nier les conditions et cadences de travail des salariés. La réalité atteint son paroxysme au Royaume-Uni et en Allemagne, où se développe la sous-traitance auprès de personnes à leur compte, confrontées à la nécessité de travailler à n'importe quel prix. Un directeur allemand d'une société de nettoyage certifie que «si le personnel de nettoyage payé par chambre n'atteint pas les quatre chambres par heure, il n'atteindra pas le salaire agréé par la convention» (VANSELow et al., 2009).
Autre trait semblable, l'hôtellerie a le taux de syndicalisation le plus bas. La représentation syndicale y est seulement de 2\% en France (contre 8\% de l'ensemble des salariés, $c f$. Amossé, 2004); elle est également peu élevée et toujours en dessous des moyennes nationales, que ce soit en Allemagne (5\%), au Royaume-Uni (4\%), au Pays-Bas (16\%) et au Danemark (25\%). Ce dernier chiffre parait singulier en Europe: en fait, l'adhésion syndicale au Danemark inclut l'affiliation à l'assurance chômage (3), ce qui explique ce taux important qui demeure néanmoins largement inférieur à celui de l'ensemble de la population active danoise (proche de $80 \%$ ). Cette faible présence syndicale peut s'expliquer par la structure et la parcellisation des hôtels sur les différents territoires, par le manque de sections syndicales spécifiques pour l'hôtellerie (et encore moins pour le personnel d'étage). Elle est également liée aux particularités des emplois avec de nombreux contrats temporaires ou à temps partiel, un turnover important, et aux caractéristiques des salariés, des femmes et des jeunes souvent d'origine étrangère, peu qualifiés, peu informés sur leurs droits, et difficilement en position de revendiquer. Au Royaume-Uni, l'individualisme des travailleurs et leur hostilité vis-à-vis des syndicats sont aussi évoqués.

Dans les pays étudiés, le pouvoir de négociation des salariés de l'hôtellerie demeure très faible, leurs syndicats étant soit absents des lieux de travail, soit impuissants du fait de la myriade des petits établissements autonomes et dispersés géographiquement. Pourtant en France un directeur d'hôtel $3 *$ signale un conflit, certes très passager: « 2 heures de grève

(3) Le Ghent système conditionne le versement de l'assurance chômage à une adhésion syndicale préalable; les employeurs danois incitent dès lors fortement leurs salariés à se syndiquer, fait unique en Europe. 
ont été menées sur la réduction du temps de travail, mais dès que les femmes de chambre ont vu qu'elles n'étaient pas payées, elles n'ont plus jamais fait grève». Aux Pays-Bas comme en Allemagne, les syndicats peuvent en principe négocier dans chaque entreprise. En fait, ce secteur est structuré dans plusieurs pays autour d'une importante et puissante union patronale représentant $40 \%$ des employeurs au Royaume-Uni, 66\% au Danemark et près de $90 \%$ en France (4). De surcroît en France, les négociations au plan national ont toujours été difficiles, dues au lobbying très développé auprès des pouvoirs publics de la part des employeurs (GUÉGNARD, Mériot, 2008). Bénéficiant depuis longtemps de nombreuses dérogations au Code du travail français (horaire, contrat, salaire hôtelier jusqu'en 2005), l'hôtellerie paraît atypique face aux autres contrées; et l'intervention du gouvernement français en faveur des hôteliers, cafetiers et restaurateurs, que ce soit en termes de temps de travail ou de financement spécifique, est plus marquée et apparaît nettement singulière ( $c f$. encadré 2$)$. La principale convention nationale collective, signée seulement en 1997, ne prévoit toujours pas de progression salariale à l'ancienneté, ni d'indemnisation des dimanches et des jours fériés travaillés (GuÉGNARD, MÉRIOT, 2005). Alors que l'entretien des chambres est majoré le dimanche aux Pays-Bas (de 1 à 3 euros de l'heure), et le week-end au Danemark pour les entreprises de nettoyage. En Allemagne, seulement le tiers des conventions collectives régionales prévoient une majoration pour les jours fériés (VANSELOW, 2008); de plus, l'association des employeurs s'oppose à l'extension sur l'ensemble du territoire allemand de la nouvelle convention collective.

\section{Une précarité plurielle}

Un même principe s'observe dans les cinq contrées. Excepté pour les personnes expérimentées, rares sont les femmes de chambre qui accèdent immédiatement à un emploi stable à plein temps, donc à la possibilité de percevoir un revenu stable et régulier. Parce qu'ils disposent d'une réserve de main-d'œuvre, la plupart des hôteliers proposent une hiérarchie de statuts entre un contrat particulièrement court ou saisonnier en bas de l'échelle (casual contract, dénommé parfois de manière évocatrice $M c J o b)$, et le contrat à durée indéterminée (regular employment). Dès lors, une segmentation apparaît nettement entre les femmes de chambre embauchées définitivement, et celles qui enchaînent les contrats éphémères. Et ce dualisme est renforcé par un second clivage entre les personnes recrutées à temps plein et celles à temps partiel. Car, autre particularité commune, les employeurs ont largement recours aux temps partiels pour gagner en flexibilité. Cela concerne près du quart des

(4) L'Union des métiers et des industries de l'hôtellerie.

\section{Encadré 2 \\ Le feuilleton du temps \\ de travail en France}

L'histoire de l'application d'une convention collective, ou le feuilleton du temps de travail, révèle au plan national des discordances entre les quatre organisations patronales, des divergences entre les cinq syndicats de salariés, et un lobbying important des employeurs envers les pouvoirs publics. L'adoption des 35 heures hebdomadaires de travail est entrée en vigueur en France le $1^{\mathrm{er}}$ janvier 2002 pour toutes les entreprises, mais l'hôtellerie-restauration a pu conserver les 39 heures.

Suite à un accord de branche signé le 13 juillet 2004 et malgré l'opposition de deux syndicats et d'une organisation patronale, le gouvernement publie l'arrêté avec effet au $1^{\text {er }}$ janvier 2005. Un syndicat, la Confédération française des travailleurs, saisit alors le Conseil d'État sur la question des horaires car cet avenant permet aux employeurs de ne pas rémunérer en heures supplémentaires des $36^{\mathrm{es}}$ aux $39^{\mathrm{es}}$ heures de travail hebdomadaires.

Le 18 octobre 2006, le Conseil d'État annule les dispositions réglementaires portant sur les 39 heures (soit le décret $n^{\circ}$ 2004-1536 du 30 décembre 2004 et l'arrêté du 30 décembre 2004). Le gouvernement réagit immédiatement en faisant voter par l'Assemblée nationale le 25 octobre 2006 un amendement pour maintenir temporairement ces 39 heures, afin d'éviter le paiement par les employeurs des heures supplémentaires, tout en appelant à un nouvel accord collectif de branche. Finalement, un avenant $\mathrm{n}^{\circ} 2$ sur le temps de travail a été signé en février 2007 par quatre syndicats et trois organisations patronales, les heures supplémentaires devront donc être payées dès la $36^{\mathrm{e}}$ heure, à partir du $1^{\mathrm{er}}$ avril 2007.

L'intervention du gouvernement, unique par rapport aux autres secteurs ou professions, est illustrée également par l'aide financière de l'État pour l'emploi de travailleurs occasionnels (notamment sous contrat d'extra) dans l'hôtellerie-restauration (décret $\mathrm{n}^{\circ}$ 2007-681 du 3 mai 2007 couvrant les périodes comprises entre le 27 décembre 2006 et le 31 décembre 2009), soit une subvention de 697 millions d'euros en 2006.

salariés en France, 44\% au Danemark, 60\% aux Pays-Bas ( $c f$. tableau 1). Plus précisément, les deux tiers des employées travaillent moins de 30 heures par semaine au Royaume-Uni. De plus, aux PaysBas, les minicontrats de 2 à 4 heures par semaine prennent de l'ampleur. Et du côté de l'Allemagne, un contraste apparaît entre le travail à temps partiel pour $6 \%$ des salariés de l'hôtellerie (contre 14\% de l'ensemble des actifs), et les minijobs (5) qui se sont fortement développés suite à une réforme

(5) En Allemagne, est défini comme minijob, un emploi dont la rémunération mensuelle est de 400 euros (ou moins), et pour lequel les employeurs ne paient que $30 \%$ des cotisations sociales. 
politique, et touchent le quart d'entre eux (contre $15 \%$ en moyenne). La gouvernante d'un hôtel $5 *$ localisé aux Pays-Bas explicite: «Pour avoir de la flexibilité nous offrons maintenant des contrats de quatre jours par semaine, de 8 heures à 15 heures hebdomadaires pour un an au départ. Par la suite, les femmes de chambre obtiennent un contrat permanent si la productivité et la qualité du travail sont suffisantes. Et nous avons une liste d'attente de personnes qui souhaitent venir travailler chez nous » (Hermanussen, 2008).

Par ailleurs, une autre différenciation oppose les entreprises. Les établissements haut de gamme (6), indépendants ou de chaîne, offrent davantage de postes permanents à plein temps pour s'efforcer de fidéliser un personnel de qualité ( $c f$. tableau 3). À l'inverse, dans l'hôtellerie économique, le personnel d'étage est majoritairement employé à temps partiel (à durée déterminée ou indéterminée). Cet éventail de contrats engendre une précarité sur le marché $\mathrm{du}$ travail, et rend ces salariées particulièrement vulnérables et dépendantes de leur employeur. La plupart des femmes de chambre interviewées espèrent obtenir un poste stable à temps plein, pour un salaire décent et une possible autonomie familiale. Comme le remarque une gouvernante néerlandaise d'un hôtel $3 *$ : «On devrait comprendre ce que signifie pour des travailleurs non qualifiés d'être condamnés à des emplois temporaires. Ils ne peuvent pas construire de futur, ils n'ont aucune sécurité» (Hermanussen, 2008).

En France, pourtant perçue comme un pays de protection de l'emploi (régulant les ruptures de contrat et le recours aux emplois temporaires), la précarité est particulièrement accrue par le contrat d'extra. Celui-ci est spécifique (7) à l'hôtellerierestauration et peut varier d'une heure à soixante jours au sein d'un même trimestre. Mais les employeurs disposent de 48 heures pour remettre les contrats d'extra aux personnes, qui sont souvent signés a posteriori ou non signés, ce qui empêche les contrôles des services de l'inspection du travail et favorise le travail au noir, notamment dans les petites structures. Il en est de même au Danemark où de nombreuses femmes de chambre peu informées de leurs droits et très peu syndiquées (le coût d'adhésion étant prohibitif), ne profitent pas de véritable protection sociale lorsqu'elles se retrouvent sans emploi. En fait, le Ghent système conditionne le

(6) Les hôtels $4 *$ et plus représentent $4 \%$ des établissements en France. Les contrastes en matière de management sont illustrés pour la France dans le tableau 3.

(7) Des contrats saisonniers, d'une durée d'un à neuf mois, se trouvent également dans ce secteur, pour effectuer des tâches censées se répéter annuellement à périodes fixes, en fonction du rythme des saisons ou des modes de vie collectifs. Le cas des travailleurs saisonniers n'a pas été rencontré dans les huit hôtels en France, les critères retenus ne prenant pas en compte le tourisme balnéaire ou de montagne. versement de l'assurance chômage à une adhésion syndicale préalable. Le Danemark organise la complémentarité de trois dispositifs habituellement faiblement coordonnés (BOYER, 2006) : le droit du travail, l'indemnisation du chômage et la politique d'activation dans l'accompagnement des demandeurs d'emploi, mais avec pour condition l'adhésion syndicale. La légendaire flexicurité danoise montre ainsi ses limites et révèle un système dual important, à travers l'exemple de l'hôtellerie: seulement $27 \%$ des salariés à temps plein bénéficient de l'assurance chômage.

Les hôteliers utilisent ces contrats précaires pour trouver une souplesse de gestion face à une activité très fluctuante, mais aussi pour effectuer une longue sélection des personnes les plus méritantes. Pour qu'une personne accède à un emploi stable, il faut attendre qu'une place se libère, donc se montrer très investie dans son travail, parfois durant plusieurs années. Cette progression au mérite devient dès lors un véritable droit au travail, puisque le nombre de chambres attribué par jour et par personne est d'abord très limité, et que seul le personnel stable de l'hôtel peut être assuré d'obtenir un revenu décent sur la base de son activité journalière. Le directeur d'un établissement $4 *$ situé dans le sud de la France avoue, comme plusieurs de ses homologues, être tenté de limiter ses recrutements sur contrats stables car «parfois, les personnes embauchées se désinvestissent un peu». Dans une zone d'emploi fortement touchée par le chômage, il préfère conserver un important volant de main-d'œuvre à sa disposition sous statut précaire, dans une situation de réelle dépendance devant accepter tout type de sollicitations et d'horaires, ou d'objectifs (en nombre de chambres à entretenir pour un temps préestimé, même si ce temps ne correspond pas à la réalité).

\section{Les risques de la sous-traitance}

L'industrie hôtelière peut choisir d'externaliser l'activité de son personnel par un recours aux agences d'intérim, ou à la sous-traitance. Dans les faits, l'intérim pour le nettoyage des chambres reste exceptionnel et limité aux situations de réelle pénurie de main-d'œuvre. Les employeurs jugent généralement cette situation coûteuse et surtout risquée, puisque l'agence d'intérim se charge simplement de sélectionner le personnel, de remplir les formalités administratives de son contrat de travail, et de le rémunérer, mais nullement d'assurer son encadrement.

En revanche, l'usage de la sous-traitance est présent, même si les statistiques restent insuffisamment fiables, et concernerait près du tiers des hôtels en France et la moitié en Allemagne, au Danemark et aux Pays-Bas. Cela permet aux hôteliers de se décharger totalement des contraintes de gestion du personnel, et des préoccupations administratives et sociales qui l'accompagnent. Les contrats de travail 
Tableau 3 : Qualité des emplois et management des femmes de chambre en France

\begin{tabular}{|c|c|c|}
\hline & Hôtels de chaîne & Hôtels indépendants \\
\hline Type de management & $\begin{array}{l}\text { Management direct. } \\
\text { Contrôles directs ou autocontrôle des } \\
\text { chambres (pour des salariées expérimentées) }\end{array}$ & $\begin{array}{l}\text { Management direct des propriétaires (ou } \\
\text { famille) } \\
\text { Contrôles directs par le propriétaire/ } \\
\text { manager }\end{array}$ \\
\hline $\begin{array}{l}\text { Utilisation d'une entreprise } \\
\text { de sous-traitance pour le } \\
\text { nettoyage }\end{array}$ & $\begin{array}{l}\text { - Pour des lieux communs (hall, salles de } \\
\text { réunion, baies vitrées...) et jardins } \\
\text { - Quelquefois pour le nettoyage des } \\
\text { chambres, à l'exception des hôtels de luxe }\end{array}$ & $\begin{array}{l}\text { Internalisation ou externalisation du } \\
\text { nettoyage des chambres et lieux communs }\end{array}$ \\
\hline $\begin{array}{l}\text { Contrats de travail: } \\
\text { - hôtels haut de gamme, de } \\
\text { luxe } \\
\text { - hôtels économiques }\end{array}$ & $\begin{array}{l}\text { - Permanents CDI à plein temps (basé sur } \\
39 \text { heures ou parfois } 37 \text { heures par semaine), } \\
\text { et quelques contrats d'extra } \\
\text { - Combinaison de contrats (CDI ou CDD } \\
\text { pour } 30 \text { heures), et un nombre croissant de } \\
\text { contrats d'extra }\end{array}$ & $\begin{array}{l}\text { Nombreux CDI temps plein et temps } \\
\text { partiel et nombreux contrats d'extra }\end{array}$ \\
\hline Salaire et autres avantages & $\begin{array}{l}\text { Salaire minimum légal } \\
\text { Un treizième mois après un an d'ancienneté, } \\
\text { assurance-maladie et retraite, participation } \\
\text { et bon d'achat, prix dans les hôtels de la } \\
\text { chaîne... }\end{array}$ & $\begin{array}{l}\text { Salaire minimum légal } \\
\text { Un rare bonus pour la fidélité ou lors d'évé- } \\
\text { nements spécifiques (Noël, canicule...) }\end{array}$ \\
\hline & Programme de formation continue & $\begin{array}{l}\text { La formation continue n'est pas une } \\
\text { priorité }\end{array}$ \\
\hline Carrière & Peu d'opportunités & $\begin{array}{l}\text { Très peu d'opportunités et aucune lorsque } \\
\text { la direction est propriétaire de l'hôtel }\end{array}$ \\
\hline Nouvelles pratiques & $\begin{array}{l}\text { - Développement d'une veille sociale et de } \\
\text { stratégies de communication } \\
\text { - Organisation d'un équilibre vie } \\
\text { professionnelle/familiale } \\
\text { - Développement de la polyvalence } \\
\text { - Introduction du travail en binôme }\end{array}$ & $\begin{array}{l}\text { Développement d'une écoute et d'un } \\
\text { soutien d'ordre personnel et social }\end{array}$ \\
\hline
\end{tabular}

Source: rapport IREDU-CEREQ, Russell Sage Foundation.

sont alors établis par la société de sous-traitance, au plus près des besoins de l'établissement, essentiellement à temps partiel(8). Les directeurs d'hôtels allemands et danois privilégient la recherche de flexibilité et la compression des coûts, comme le cost-cutting strategy en Allemagne (VANSELow, 2008). D’ailleurs, un responsable allemand remarque que «le budget est devenu le mot magique» et le principe du paiement par chambre est un bon argument commercial (le tarif varie de 1,6 à 3 euros par chambre) dans les sociétés de sous-traitance. Et au Danemark, la différence de prix est faible, entre 14,60 à 14,90 euros de l'heure pour une société externe de nettoyage (contre 13 à 14,30 euros en interne).

A contrario, des managers français, plutôt favorables à l'internalisation du nettoyage des chambres, défendent l'idée de la personnalisation du service, du suivi de la qualité, de la fidélisation du personnel... Une directrice adjointe d'un hôtel 2 * de chaîne justifie ainsi un tel choix : «On a décidé d'internaliser la gestion du personnel des étages

(8) En France, ces contrats oscillent entre 20 et 30 heures hebdomadaires; et le tiers des salariés des entreprises de nettoyage travaillent à temps partiel contre le quart de ceux de l'hôtellerie. (Source: Enquêtes Emploi 2000-2002). parce qu'au niveau de la propreté, une femme de chambre qui appartient à l'hôtel s'investit plus qu'une femme de chambre extérieure à l'hôtel». En fait, Accor, leader de la chaîne hôtelière a opéré un net recul en France dans son recours à la soustraitance suite à un problème d'image commerciale, après d'importants conflits sociaux survenus au sein d'une entreprise sous-traitante (PuECH, 2004)(9). Cette lutte ou grève atypique a été un élément déterminant pour Accor qui a depuis internalisé une partie de l'entretien de ses hôtels, et embauché les anciennes employées des sociétés de nettoyage. De même, les hôteliers néerlandais préfèrent fidéliser leur personnel, 1'heure de sous-traitance étant chère (8 à 9 eurospar chambre) et de moindre qualité; un directeur d'hôtel $3 *$ confirme: «Un cheveu dans une salle de bain peut entraîner des dommages à la réputation d'un hôtel» (HERMANUSSEN, 2008).

(9) En 2002, le personnel de cette société de nettoyage s'était mis en grève pour améliorer ses conditions et cadences de travail. Les femmes de chambre salariées dans les hôtels du groupe nettoyaient seize chambres en 8 heures, alors que les employées de la société de nettoyage devaient en nettoyer vingt à vingt-trois en 6 heures (en étant payées 7,16 euros de l'heure, soit 49 centimes de plus que le SMIC). 
Si la sous-traitance se développe différemment selon les pays, ils se rejoignent tous sur un point: les cadences de travail des salariées d'entreprises sous-traitantes sont supérieures à celles des personnels gérés en direct par les hôteliers. Une ancienne employée d'une société de nettoyage embauchée actuellement dans un hôtel de chaîne $2 *$ témoigne de son expérience: "Nous devions faire quatre chambres et demie à l'heure. C'était impossible. En fait, pour préparer quatre chambres, on travaillait 1 heure ou 2 de plus chaque jour, qui n'étaient pas payées. Alors ici c'est le paradis ici franchement. Je ne regrette pas. Au début, je ne voulais pas venir. J'ai dit plus jamais femme de chambre, plus jamais des hôtels». Là encore, les directeurs d'hôtels haut de gamme se distinguent et tendent à limiter ce recours à la sous-traitance. Ils adoptent davantage une administration directe et une fidélisation des salariées en offrant des contrats stables à plein temps. De plus, la personnalisation du service est important, notamment quand des clients insistent pour être à tel étage afin d'avoir le service de telle femme de chambre, comme l'affirme un manager français d'un hôtel $3^{*}$ : «Face à un marché hôtelier compétitif au niveau qualité prix, le charme joue».

Au final, l'hôtellerie n'est jamais apparue pionnière en matière d'avancées sociales dans aucun de ces territoires. La prédominance et la dispersion des petites unités, le pouvoir des organisations d'employeurs, la faiblesse de la présence des syndicats au niveau local, sont autant de freins à la régulation du marché du travail, quel que soit le pays. L'inégal accès à un poste stable à plein temps souligne un dualisme pluriel croissant et les pratiques des employeurs accentuent la segmentation sur le marché du travail. De telles stratégies ne sont pas sans répercussion sur la qualité des modes de vie au quotidien et les perspectives offertes aux femmes de chambre, exposées dans les pages suivantes.

\section{Le labeur des femmes de chambre}

Les femmes de chambre présentent des particularités discriminantes sur le marché du travail qui se retrouvent dans les cinq pays européens: une population féminine (car les rares valets de chambre n'effectuent pas les mêmes tâches), âgée de quarante ans en moyenne, fréquemment mère de famille, peu diplômée, la plupart de nationalité ou d'origine étrangère. Les employeurs sélectionnent leur personnel sur ces caractéristiques individuelles et ethniques pour occuper ces emplois du marché secondaire (Reich et al., 1973). Les femmes de chambre, qui représentent entre $20 \%$ et $30 \%$ des salariés de l'hôtellerie, tiennent une place particulière: une position subalterne au sein des employés, une invisibilité visà-vis de la clientèle, une activité pénible et servile, un manque de perspectives professionnelles.

\section{Une main-d'œuvre cosmopolite}

Autre exemple de similarité, les directions font état d'un recrutement plus aisé et d'un turnover plus faible que pour les autres professionnels de l'hôtellerie. Les femmes de chambre forment un groupe composite mis en lumière par l'hétérogénéité de leur profil. Selon les pays et les hôtels, entre $20 \%$ et $60 \%$ des femmes de chambre sont nées à l'étranger (cf. tableau 2). Les origines ethniques peuvent être très variables et diffèrent selon les lieux (10) de travail, les années et la main-d'œuvre locale disponible. Ainsi, les hôtels de Londres qui privilégièrent longtemps les migrantes venant du Commonwealth, embauchent maintenant des personnes de Pologne (DutTon et al., 2008). La cooptation est une pratique courante, certains hôtels emploient massivement des Asiatiques ou des Portugaises, des femmes venant du Maghreb ou d'Afrique, d'autres embauchent des personnes des pays d'Europe de l'Est. Une différenciation apparaît dans deux territoires: Amsterdam attire davantage la main-d'œuvre étrangère, de plus en plus masculine notamment dans les entreprises de nettoyage, alors que les hôtels de province des Pays-Bas recrutent surtout des Néerlandaises (Hermanussen, 2008). La majorité des femmes de chambre sont des immigrées en Allemagne de l'Ouest, alors qu'elles sont de nationalité allemande en Allemagne de l'Est, là où la main-d'œuvre étrangère est rare et le chômage élevé (VANSELOW, 2008).

Mais au-delà de ces caractéristiques sociales, une spécificité commune à l'ensemble des femmes de chambre est la nécessité pour elles d'accepter un tel poste, quelle que soit la pénibilité de l'activité professionnelle. Une femme de chambre dans un hôtel français $4 *$ précise: "Franchement, le travail de femme de chambre n'est pas un boulot qui plaît forcément. Pour moi il faut obligatoirement travailler et il faut faire ce que tu peux. Moi par exemple, je n'ai pas de diplôme donc... forcément il faut travailler, donc j'ai choisi ça. Si je trouve mieux, c'est sûr que je pourrais laisser femme de chambre, ce n'est pas toujours facile. C'est un boulot très dur et physique». Ce métier est souvent choisi par défaut face à l'impossibilité d'exercer une autre activité, et relève plutôt du qualificatif femme de ménage. Ceci est confirmé par un directeur d'hôtel $3^{*}$ de Glasgow: "Ce sont des femmes qui fondent une famille lorsqu'elles sont jeunes, elles ont alors besoin de travailler à temps partiel pour gagner de l'argent et elles s'y habituent et quand elles arrivent à 50 ans, elles ne pensent pas à évoluer, elles ne pensent pas qu'elles peuvent faire autre chose» (DuTTON et al., 2008). Les employées ont en commun d'être captives de cet emploi, d'autant qu'un grand nombre d'entre elles parlent difficilement la langue du pays.

(10) La part des femmes de chambre d'origine étrangère peut ainsi varier de $0 \%$ dans un hôtel de Glasgow à $100 \%$ dans un hôtel de Londres. 
De plus, aucune qualification n'est véritablement requise. Comme le souligne une gouvernante d'un hôtel $4 *$ en France : «La personne doit être sérieuse, discrète et la propreté est un critère essentiel. Une femme de chambre n'a pas de compétences particulières à avoir, elle doit juste savoir utiliser les codes de couleur, savoir lire les chiffres. » Dans de nombreux hôtels, les femmes de chambre ont des supports récapitulatifs avec des codes couleurs pour se repérer (en bleu les chambres de départ à faire entièrement...). Le nettoyage est une activité qui s'apprend rapidement, sur le tas, et «Vous n'avez pas besoin de compétences pour faire la poussière ou passer l'aspirateur» déclare une femme de chambre d'un hôtel haut de gamme au Royaume-Uni. La formation à l'exercice du métier s'effectue généralement sur le tas, par observation, apprentissage, imitation ou shadowing d'une collègue, en un à deux jours maximum selon la catégorie d'hôtel. À partir du troisième jour, il est attendu une grande autonomie professionnelle et une rapidité d'exécution comparable à celle des personnes expérimentées. Rares sont les femmes de chambre qui possèdent un diplôme de l'hôtellerie. La majorité des salariées ne possèdent aucun diplôme ( $c f$. tableau 2), quelquesunes ont suivi une formation non reconnue dans le pays d'accueil; elles sont aisément fidélisées du fait de leur faible employabilité sur le marché du travail.

\section{Rythme et tempo de l'activité}

Les femmes de chambre ont en charge la propreté, l'ordre et le confort des chambres de l'hôtel et quelquefois des parties communes ou de la réception. Elles entretiennent quotidiennement entre sept et vingt et une chambres en moyenne, au rythme de 15 à 45 minutes par chambre selon la catégorie d'hôtel ( $c f$. tableau 2). Ce quota tend à diminuer dans l'hôtellerie de luxe. Leur nombre varie non seulement en fonction de la capacité hôtelière, mais aussi selon la classification, la typologie des chambres (duplex, suite, chambre VIP...), l'éloignement des chambres ou le nombre d'étages, la présence d'offices à chaque étage, la possibilité de déléguer l'entretien des parties communes à un autre personnel, ou les contrôles à une gouvernante... Une pratique d'autocontrôle est de plus en plus exigée, mais certains hôtels haut de gamme optent pour le renforcement des équipes de gouvernantes afin d'éviter tout risque de non-qualité, face à un personnel peu qualifié et en présence d'une offre hôtelière concurrente importante.

De fait, la nature du travail et le rythme imposé pèsent sur l'activité des femmes de chambre qui doit s'effectuer en l'absence des clients, afin de ne pas déranger par leur présence: elles doivent être aussi discrètes que la poussière. Au Royaume-Uni, une gouvernante compare même son personnel «aux elfes dans Harry Potter où les lits sont faits, le travail est effectué, mais personne ne les voit» (in DutTon et al.). Les tâches diffèrent peu d'un pays à l'autre et n'ont guère évolué au fil des ans : nettoyage et mise en ordre de la chambre selon des standards et procédures définis par l'hôtel ou la chaîne. Le poste comporte surtout des opérations techniques: de l'agencement des chambres au nettoyage, éventuellement entretien du linge, tests d'éclairage ou de chauffage, disposition des produits, activités de maintenance de base, gestion des chariots et stocks (linge, produits)... Il comprend aussi des fonctions de coordination et de gestion, tant au sein de l'équipe qu'avec les autres services, voire avec la clientèle, et parfois la formation des nouvelles arrivantes. Certes, toutes les femmes de chambre n'accomplissent pas l'ensemble de ces activités: dans le domaine de l'organisation du travail et des relations à la clientèle par exemple, certaines responsabilités concernent exclusivement la hiérarchie. De plus, l'hôtellerie haut de gamme requiert davantage de technicité et de savoir-être. Néanmoins, la multiplicité des tâches et la pénibilité du métier, par ailleurs faiblement rémunéré, représentent l'ultime paradoxe de cet emploi marginalisé.

Dans la plupart des hôtels européens visités, les femmes de chambre restent isolées, peu intégrées à la vie collective de l'établissement, cantonnées dans les étages à passer de nombreuses heures seules ou à ne croiser que leurs collègues ou les gouvernantes. «C'est un travail très solitaire» confie une gouvernante anglaise. Cet isolement est souvent vécu de manière positive, de la part des salariées, comme un sentiment d'autonomie ou d'indépendance. Mais, cette liberté reste toute relative du fait des contraintes de temps accordé par chambre et des standards appliqués dans les hôtels. Des exceptions confirment la règle. Ainsi dans des hôtels de province aux Pays-Bas, le nettoyage des chambres s'effectue en équipe de deux, voire trois personnes; et en France, quelques hôtels de chaîne haut de gamme limitent la pénibilité et l'isolement par la possibilité de travailler en binôme.

Pour autant, le rythme de travail des femmes de chambre est soutenu, éprouvant, et tous les managers s'accordent pour affirmer que ce métier est physique et fatigant, le plus ingrat, pas assez valorisé, pas très connu, or c'est aussi disent-ils, «l'image de l'hôtel», «le métier caché», «les cartes de visite de l'hôtel». L'analyse menée par la Fondation européenne pour l'amélioration des conditions de vie et de travail (EUROPEAN FOUNDATION FOR THE IMPROVEMENT OF LIVING AND WORKING CONDITIONS, 2004) rappelle la difficulté physique du métier (se pencher, se redresser ou s'accroupir en permanence), la nécessité de soulever de lourdes charges (pour faire les lits ou déplacer du mobilier) et le contact permanent avec l'eau ou les produits d'entretien (qui peuvent créer des allergies ou des infections biologiques). L'usure physique, et particulièrement les maux de dos, ou les allergies aux produits d'entretien, représentent les troubles fréquemment cités. Parmi les tâches jugées pénibles figurent le nettoyage des grands miroirs ou des 
baignoires, qui imposent des postures difficilement supportables, des sols sombres en marbre, et depuis peu la confection de grands lits, king size, dotés de couettes et de matelas de plus en plus épais, utilisés comme argument commercial. Un autre élément d'intensification du travail se réalise à travers la politique de qualité des prestations apportées aux clients: des peignoirs, davantage de produits, de commodités et de services au niveau de la chambre. Tout ceci entraîne une intensité du travail à effectuer dans un délai court, comme le signale une gouvernante britannique d'un hôtel haut de gamme: «Ce n'est pas seulement physique, c'est aussi mental, car vous avez besoin de vous souvenir de chaque chose à faire» (DutTon et al., 2008).

\section{“Il faut speeder"}

Le personnel a un planning pour la journée, communiqué le matin ou au fur et à mesure des départs, parfois avec un ordre de priorité ou en réponse aux attentes de la clientèle. Quelquefois, certains managers en profitent pour fixer des objectifs irréalisables. Une femme de chambre, embauchée depuis deux ans dans un hôtel $4^{*}$ après trois années de travail en «extra», explique: «Des fois il faut speeder. On nous dit d'aller plus vite. Moi je trouve que les 30 minutes par chambre qu'on nous donne, pour un $4 *$, ce n'est pas juste. Parce que pour qu'une chambre soit bien faite, il faut minimum 40 minutes. Parce que je décide souvent de faire au moins une ou deux chambres à fond, pour bien que tout soit fait... 30 minutes, c'est... Pour seulement faire la poussière et bien aspirer en bas des lits, parfois tu fais 20 minutes». Ceci se retrouve également du côté des hôtels économiques et une salariée française qui y travaille depuis dix ans affirme: «Il n'y a pas assez d'heures donc on est obligé de speeder pour qu'on soit à l'heure... mais on a un manque de temps, on n'y arrive pas ».

Autre fait commun: l'organisation du temps de travail apparaît comme la résultante des contraintes concurrentielles et organisationnelles essentiellement construites par les employeurs (GADREY, 2002), notamment les chaînes qui appliquent une standardisation des tâches. La cadence de travail est toujours soutenue, éprouvante, et le stress des femmes de chambre paraît également comme un élément crucial analogue aux cinq pays face à l'intensification de l'activité. Ainsi au Royaume-Uni, ou en France, de nombreuses salariées ne peuvent bénéficier des pauses réglementaires, du fait de la pression des employeurs. Une des principales sources d'inquiétude est également la découverte de l'état de la chambre. Le directeur d'un établissement haut de gamme en France précise qu'elles «ont le stress de ne pas tenir le rythme face à l'objectif temps et le nombre de chambres». Aux PaysBas, la gouvernante d'un hôtel $4 *$ atteste: «Quand j'ai commencé ce travail en 2000, j'avais 3 minutes de plus par chambre. Maintenant nous faisons le même nombre et parfois plus de chambres en moins de temps, mais la limite est atteinte, je n'accepterai pas davantage de réduction de temps, nous sommes des êtres humains, pas des robots» (Hermanussen, 2008). Un directeur d'hôtel $3 *$ au Royaume-Uni souligne également la routine de l'activité: «J'ai vu cette femme faire vingt chambres. Nettoyer vingt chambres est si impressionnant, si difficile, que c'est ce que j'appelle un travail de routine, vous savez, comme dans le film Groundhog Day, la journée sans fin de la marmotte: chaque jour se ressemble et le travail recommence, chaque chambre est salie de la même façon, il faut la remettre en ordre selon le même standard» (DutTon et al., 2008).

\section{Un faible espoir d'évolution}

Parmi les indicateurs retenus dans l'analyse de la qualité de l'emploi, figurent la formation et le développement de la carrière (DAVOINE, ERHEL, 2007). En fait, la formation est quasi inexistante pour les femmes de chambre dans les petits hôtels indépendants. Seuls quelques-uns des hôtels visités proposent des actions à destination du personnel d'étages : journées d'information et de formation aux postures ergonomiques, produits et techniques de nettoyage, accueil de tels clients étrangers dans les hôtels de luxe, sensibilisation à la satisfaction du client... Quelques hôtels de chaîne de catégorie modeste et certains petits établissements indépendants favorisent une évolution professionnelle en développant l'apprentissage d'un nouveau métier(11) notamment vers le service ou la réception (GUÉGNARD, 2004). En fait, pour progresser dans la carrière, il faut faire preuve d'une disponibilité importante et accepter éventuellement une forme de polyvalence pour répondre aux besoins de l'hôtel. Il faut aussi avoir une excellente maitrise d'une ou plusieurs langues.

En réalité, les perspectives professionnelles demeurent restreintes pour les femmes de chambre, compte tenu de leur âge et de leur faible qualification, avec souvent la barrière de la langue. Les possibilités de promotions internes sont restreintes et symboliques, et se limitent à l'accès aux postes de première femme de chambre, d'aide gouvernante puis, de gouvernante uniquement dans les grands établissements, s'appuyant dans les chaînes sur une mobilité géographique incompatible avec des contraintes familiales. La taille réduite de nombreux hôtels et la présence des propriétaires à la tête des établissements familiaux brisent toutes perspectives. Un poste à plein temps représente souvent le seul espoir d'aboutissement de leur carrière, comme l'exprime vivement une femme de chambre embauchée depuis cinq ans dans un hôtel français $2 *$ : «J'aimerais bien travailler à temps complet [...]. On l'a demandé, mais pour l'instant ce n'est pas possible».

(11) Exemple, la démarche Acteurs 2003 dans les hôtels Ibis en France (GUÉGNARD, 2004). 
Plusieurs personnes déplorent leur situation précaire, à l'instar de cette employée qui se qualifie d' "extra fixe». Son histoire est la suivante: arrivée en France il y a douze ans, elle a suivi deux mois de formation pour pouvoir parler le français. Engagée dans une société de nettoyage comme femme de ménage pour plusieurs employeurs, elle cumulait jusqu'à 13 heures de travail par jour. Par la suite, elle a effectué un stage de formation professionnelle de femme de chambre et malgré son diplôme est embauchée depuis quatre ans sous statut d'extra dans un hôtel $4^{*}$ : «Quand on est extra, il faut se battre tous les jours, on fait ce qu'on peut». Il faut également que les femmes de chambre demeurent disponibles à tout prix: une gouvernante d'hôtel $3^{*}$ demande clairement à ses extras d'éviter de s'engager ailleurs. Elles sont alors contraintes de se maintenir dans la situation aléatoire qui leur est offerte, au risque de manquer d'autres opportunités professionnelles.

L'organisation du travail et la conciliation de la vie professionnelle et familiale sont évoquées dans seulement trois pays. En Allemagne, les personnes interviewées sont satisfaites de leur planning, arrivent à concilier leurs activités professionnelles et la famille, même si elles travaillent le week-end; et quelques conventions régionales leur donnent entre dix à quinze dimanches non travaillés par an. Au Danemark, le travail (de 5 à 6 heures par jour) est effectué au moment où les enfants sont à l'école, et ceci permet aux mères de passer davantage de temps dans leur foyer. Alors qu'en France, la contrainte du week-end est parfois jugée difficile quand elle interfère avec la vie de famille, d'autant plus que la convention collective de l'hôtellerie ne prévoit pas de majoration pour le travail des jours fériés et des dimanches. À cette astreinte, s'ajoute celle de la localisation du travail. Le personnel réside rarement dans la capitale du pays ou à proximité des hôtels prestigieux et doit consacrer un temps important à ses déplacements (par exemple, d'1 heure à une 1 heure et demie par trajet à Paris). En province, les hôtels de chaîne parfois situés hors de la zone urbaine posent les difficultés des transports en commun, pas toujours bien desservis, en particulier les samedis et dimanches. Cette contrainte s'ajoute à la lourde charge physique de leur activité, avec des déplacements tôt le matin et tard le soir.

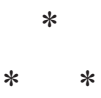

Entre précarisation et subordination, la qualité de l'emploi des femmes de chambre est identique dans les cinq pays étudiés, tant en termes de conditions d'emploi (contrat, rémunération, durée et sécurité, formation continue, carrière...), que d'activités (horaires, cadence, intensité, compétences...). Le type d'hôtel et le temps de travail se combinent pour créer des inégalités, diversifier les statuts, et enfermer certaines dans un monde précaire. Un clivage apparaît en fonction des catégories d'établissement. Par exemple, l'hôtellerie de luxe (au Danemark, au Royaume-Uni ou aux Pays-Bas) offre plutôt de meilleures conditions d'emploi, avec parfois même des bénéfices financiers (primes exceptionnelles) ou en nature. Il existe également des avantages dans les hôtels de chaîne en Allemagne et en France, avec de meilleures situations de travail et un respect de la législation (notamment des horaires, des congés, avec un treizième mois, une mutuelle, quelques formations...), qui fait souvent défaut dans l'hôtellerie indépendante. Dans ces hôtels, le sort des femmes de chambre apparait plus enviable, mais les avancées restent rares et partielles, et la menace du recours à la sous-traitance est toujours en filigrane.

Dans les cinq contrées, les femmes de chambre, isolées, peu qualifiées et mal informées, n'ont guère de ressources pour contrer une intensification de l'activité ou une application fantaisiste de la législation. Vulnérables et peu exigeantes, la majorité des salariées sont aisément fidélisées du fait de leur faible employabilité sur le marché du travail. Elles ont en commun d'être captives face à cette situation, d'autant qu'un grand nombre d'entre elles peinent à s'exprimer dans la langue de leur pays d'accueil. Elles occupent des emplois du marché secondaire qui, selon la théorie du marché dual, sont peu rémunérés et particulièrement difficiles (stress, cadences élevées, délais courts), offrant des chances de promotion quasi inexistantes. Les entreprises hôtelières adoptent donc des comportements identiques dans des contextes nationaux distincts: la qualité des emplois proposés diffère peu, malgré un cadre institutionnel propre à chaque pays (lois, normes, conventions...), mais ce sont leurs pratiques de gestion de main-d'œuvre qui accentuent la segmentation du marché du travail.

De ce fait, les femmes de chambre connaissent des destinées similaires malgré des contextes institutionnels différents. Elles conquièrent généralement leur emploi sur la durée, parfois heure par heure, jour après jour. La plupart d'entre elles débutent leur parcours par des contrats éphémères et irréguliers, avec des temps partiels non choisis. Elles subissent à la fois l'insécurité du travail et tous les mauvais côtés de la flexibilité, même au Danemark. Et, avec le temps, lorsque la fatigue s'installe, les plus expérimentées se trouvent dans l'obligation de réduire progressivement leur rythme ou leur journée: soit en travaillant de nouveau à temps partiel, soit en cherchant une activité hors de l'hôtellerie, ce qui bien souvent les reconduit vers la précarité ou le chômage. Ainsi, au lieu de développer par le travail leurs opportunités de professionnalisation et d'accès à la reconnaissance patronale, elles s'exposent à un risque médical d'usure professionnelle puis d'exclusion sociale. Leur expérience peut donc se retourner contre elles. 
Les destins analogues des femmes de chambre sont fortement empreints des stratégies organisationnelles du secteur de l'hôtellerie, et même directement marqués par la politique d'efficacité de certains dirigeants hôteliers. Ces derniers adoptent le principe d'un travail aux objectifs au risque de creuser l'écart entre le temps travaillé et le temps rémunéré. La caractéristique domestique de l'activité facilite d'ailleurs l'instauration de rapports de dépendance, vis-à-vis du directeur ou d'un simple membre de la hiérarchie, que ce soit dans des micro-entreprises familiales, des hôtels importants, ou des établissements affiliés à une chaîne. Ainsi, les femmes de chambre dépendantes des pratiques organisationnelles et gestionnaires de leurs employeurs dans les cinq pays d'Europe, se trouvent confinées dans des emplois sans perspectives, non pérennes et même à risques sur la durée. Elles peuvent alors apparaître simultanément les bénéficiaires et les victimes des politiques publiques qui encouragent la flexibilité des emplois.

\section{Bibliographie}

Amossé T. (2004), «Mythes ou réalités de la syndicalisation en France», Premières Synthèses, Premières Informations, $\mathrm{n}^{\circ} 44.1$, Dares, Ministère de l'emploi, du travail et de la cohésion sociale, octobre.

Appelbaum E., Bernardt A., Murname R.J. (eds) (2003), Low Wage America: How Employers are Reshaping Opportunity in the Workplace, Russell Sage Foundation, New York.

Beauvois M. (2003), «L'hôtellerie, la restauration et les cafés, un secteur très spécifique en termes d'emploi et de rémunération », Insee Première $n^{\circ} 889$, mars.

Boyer R. (2006), La flexicurité danoise, quels enseignements pour la France?, collection du Cepremap, Paris, Éditions rue d'Ulm.

Caroli E., Gautié J. (eds) (2008), Low-Wage Work in France, Russell Sage Foundation, New York.

Caroli E., Gautié J. (sous la direction de) (2009), Bas salaires et qualité de l'emploi: l'exception française?, collection du Cepremap, ${ }^{\circ} 17$, Paris, Éditions rue d'Ulm.

Doeringer P.B., Piore M.J. (1971), Internal Labor Markets and Manpower Analysis, Heath Lexington books, Lexington, Massachusetts.

Dutton E. et al. (2008), "Just like the Elves in Harry Potter: Room Attendants in UK Hotels", in C. Lloyd, G. Mason, K. Mayhew (eds), Low-Wage Work in the United Kingdom, Russell Sage Foundation, New York.

ERIKSSON T., Li J. (2008), "Restructuring Meets Flexicurity - The case of Danish Room Attendants", in N. Westergaard-Nielsen (ed.), Low-Wage Work in Denmark, Russell Sage Foundation, New York.

EUROPEAN FoUNDATION FOR THE IMPROVEMENT OF LIVING AND WORKING CONDITIONS (2004), EU hotel and restaurant sector: Work and employment conditions.

Eurostat (2007), Tourism statistics, European Commission, Pocket Books.

Gadrey J. (sous la coordination de), Djellal F., Gallouj C., Jany-Catrice F., Mériot S.-A., Ribault T. (2002), Hôtellerie-restauration: héberger et restaurer l'emploi (les cas français, américain et japonais), Paris, La Documentation française.
GuÉGNARD C. (sous la coordination de) (2004), «À la recherche d'une conciliation des temps professionnels et personnels dans l'hôtellerie-restauration ", Relief $\mathrm{n}^{\circ} 7$, Céreq, septembre.

Guégnard C., Mériot S.-A., Bosse N., Roualdès D. (2005), French Hotel Industry: Traditions and Social Developments, report for Russell Sage Foundation, March.

Guégnard C., Mériot S.-A. (2007), «Les emplois à bas salaire et les salariés à l'épreuve de la flexibilité», Bref $\mathrm{n}^{\circ} 237$, Céreq, janvier.

GuÉGnard C., Mériot S.-A. (2008), "Housekeepers in French Hotels: Cinderella in the Shadow", in E. Caroli, J. Gautié (eds), Low-Wage Work in France, Russell Sage Foundation, New York.

GuÉGnArd C., Mériot S.-A. (2009), «Alice au pays des hôtels: de l'autre côté du miroir», in E. Caroli, J. Gautié (sous la direction de), Bas salaires et qualité de l'emploi : l'exception française?, collection du Cepremap, $\mathrm{n}^{\circ} 17$, Paris, Éditions rue d'Ulm.

Hermanussen R. (2008), “Hotels : Industry Restructuring and room attendants" in W. Salverda, M. Van Klaveren \& M. Van der Meers (eds) Low-Wage Work in the Netherlands, Russell Sage Foundation, New York.

Piore, M.J. (1978), "Dualism in the labour market. A response to uncertainty and flux. The case of France" Revue Économique, vol. 19 n¹, 26-48.

Puech I. (2004), «Le temps du remue-ménage. Conditions d'emploi et de travail des femmes de chambre», Sociologie du travail, avril-juin 2004, 46, n 2 .

Reich M., Gordon D.M., Edwards C. (1973), “A theory of Labor Market Segmentation", American Economic Review, Vol. 63, n² 2, May.

VANSELOW A. (2008), "Still lost or forgotten? The work of hotel room attendants in Germany" in G. Bosch and C. Weinkopf (eds) Low-Wage Work in Germany, Russell Sage Foundation, New York.

Vanselow A., Warhust C., Bernhardt A., Dresser L. (2009), Hotel Room Attendants and Job Quality: A comparison of Work, Wages and Labour Market Institutions in Europe and the US, paper to the 27th Annual International Labour Process Conference, Edinburgh. 